\title{
Trypsin digest of cancer cells surface stimulates anti-tumor immune response better than cancer cells themselves
}

\author{
Elena E. Balashova and Petr G. Lokhov
}

ZAO BioBohemia, Moscow, Russia

Correspondence should be addressed to Petr G. Lokhov, e-mail: lokhovpg@rambler.ru

\begin{abstract}
Antigens expressed on the surface of cancer cells are accessible targets for both humoral and cell-mediated immune responses, and are therefore potential candidates for vaccine development. Treating surface of live human breast adenocarcinoma cells (MCF-7) with trypsin yields a digest that contains $0.7 \%$ of total cell protein. Despite this difference, the trypsin digest stimulates in cytotoxicity assays anti-tumor response which kills $10-40 \%$ more cancer cells than those stimulated with cells themselves. From these results, we concluded that trypsin digest obtained from live cancer cells contains the essential antigens to induce an immune-mediated anti-tumor effect, and therefore, is candidate for anti-tumor vaccine development.
\end{abstract}

Key words: cellular cancer vaccine, tumor-associated antigens, cell surface antigens

\section{Introduction}

Vaccines which use cancer cells as a source of these antigens have been investigated for more than 20 years, and cell-based vaccinations show great potential for prevention different types of cancers (1). Furthermore, cell-based vaccinations have an advantage over other immuno-therapies that target only specific epitopes since cell-based vaccines can recognize multiple native antigens, many of which have not been isolated or characterized (2). However, there are several aspects of cell-based vaccinations that require further development. For example, while cancer cells provide surface antigens that are targets for a desired immune response, they also contain a high abundance of house-keeping proteins, carbohydrates, nucleic acids, and other intracellular contents that are ubiquitous in mammalian cells. These ubiquitous molecules are undesirable for targeting immune-based therapies. Another potential limitation with cell-based vaccines is the difficulty of purifying cells from possible dangerous intracellular contaminants such as cellular parasites, viruses, toxins and prions. Improving these aspects of cellbased vaccines is essential for improving the stringency and efficacy of cancer vaccines (3-4).
One possible and attractive method to improve the development of cancer vaccines is to isolate essential targets of tumor cells which can be used to develop vaccines. The meaningful targets for immune responses are mainly domains of surface-expressed proteins and glycoproteins that are accessible to antibodies and cytotoxic immune cells. (57) These targets are also accessible to proteases, and therefore they can potentially be isolated by proteolytic cleavage (8). The antigen profile obtained by proteolytic cleavage contains a comprehensive set of native antigens that can be used to direct immuno-therapeutics while minimizing the amount of ubiquitous molecules that are not useful targets. Improving methods of antigen isolation could potentially improve the efficiency with which cancer vaccines can be developed.

This work shows that proteolytically-cleaved cell surface antigens from live tumor cells can be used to stimulate immune responses directed toward same tumor cells. For this purpose the trypsin digest from live human breast adenocarcinoma cells (MCF-7) was used to stimulate immune-mediated cytotoxic responses against MCF-7 cells in vitro. 


\section{Materials and Methods}

\section{Preparation of lysate and trypsin digest from MCF-7 cells}

MCF-7 cells (ATCC) were grown to 75\% confluence in RPMI-1640 (PanEco, Russia) supplemented with $10 \%$ fetal bovine serum (FBS). The cells were washed $5 \times$ with Hank's Balanced Salt Solution (HBSS) and trypsin $(0.2 \mu \mathrm{g} / \mathrm{mL}$, activity $15000 \mathrm{U} / \mathrm{mg}$, Promega) solution prepared on HBSS was added to the cells $\left(40 \mu \mathrm{L} / \mathrm{cm}^{2}\right)$. Cells were incubated at $37{ }^{\circ} \mathrm{C}$ in saturated humidity for $20 \mathrm{~min}$. Trypsin solution was collected and centrifuged $(600 \times g$ for $5 \mathrm{~min})$. The resulting supernatant (denoted as trypsin digest) contained cell surface protein fragments and trypsin. Cell viability after treatment with trypsin was determined directly in culture flask using the trypan blue exclusion method (9). MCF-7 lysate was prepared as previously described (10). The protein concentration of the lysate and trypsin digest was determined with $\mathrm{BCA}^{\mathrm{TM}}$ Protein Assay Kit (Thermo Scientific, USA).

\section{Preparation of tumor lysate- and trypsin digest-loaded DC}

Dendritic cells (DC) were generated as described previously (11). Briefly, fresh peripheral blood mononuclear cells (PBMC) from healthy donors were isolated by Ficoll-Hypaque gradient centrifugation and then allowed to adhere to culture flasks for $1 \mathrm{~h}$. The fraction of non-adherent cells was collected and centrifuged. Cell pellets were mixed with autologous serum containing $10 \%$ DMSO and stored in liquid nitrogen. Cryo-preserved non-adherent PBMC were later used as a source of effector cells (cytotoxic $T$ lymphocytes, CTL) for cytotoxicity assays. The adherent cell fraction was harvested and cultured in RPMI-1640 supplemented with $10 \%$ FBS in the presence of $1000 \mathrm{U} / \mathrm{mL}$ granulocyte macrophage colony-stimulating factor and $1000 \mathrm{U} / \mathrm{mL}$ interleukin-4 (Sigma-Aldrich). Six days after culture, either MCF-7 cell lysate (final concentration of $270 \mu \mathrm{g}$ of protein $/ \mathrm{mL}$ ), or trypsin digest (2 $\mu \mathrm{g}$ of protein $/ \mathrm{mL}$ ) was added to equal volume of immature DC. After $3 \mathrm{~h}$ of incubation, DC were matured with $1000 \mathrm{U} / \mathrm{mL}$ of tumor necrosis factor-a (Sigma-Aldrich) for $48 \mathrm{~h}$. For successive cycles of CTL stimulation, aliquots of lysate- or trypsin digest-loaded DC were cryo-preserved and thawed as needed. The freezing method used was described previously (12).

\section{Stimulation of CTL}

Lysate- or trypsin digest-loaded DC $(4.5 \times$ $10^{4}$ ) were combined with $9 \times 10^{5}$ autologous non-adherent PBMC $(1: 20)$ in 4 $\mathrm{mL}$ of RPMI-1640 medium supplemented with $30 \mathrm{U} / \mathrm{mL}$ of clinical grade human interleukin-2 (Ronkoleukin, Russia) and $10 \%$ FBS. The culture medium supplemented with interleukin-2 was replaced every third day. The next portion of antigen-loaded DC was added to PBMC seven days after stimulation. After next five days, the non-adherent PBMC containing stimulated CTL were washed by centrifugation and used in cytotoxicity assays as effector cells.

\section{Cytotoxicity assay}

MCF-7 cells were seeded at $5 \times 10^{3}$ cells/well into 48-well culture plates, and after $48 \mathrm{~h}$ of incubation cultures had expanded to $5 \times 10^{4}$ cells/well. Effector CTL were then added to MCF-7 cells at specific effector:target ratios. At each time point, wells were washed to remove $C T L$, attached MCF-7 cells were trypsinized and measured for viability by trypan blue exclusion. Cell counts were averaged over three measurements, and the number of surviving MCF-7 cells was plotted against time. The killing rate per day per effector CTL is derived from the difference in rate constants on the log linear plots of MCF-7 cell growth curves in the presence and absence of effector CTL (13).

\section{Results}

Calculation of dead cells in microscopic fields showed that cell death rate does not exceed $0.5 \%$ by treating cells with trypsin for $20 \mathrm{~min}$ (Fig. 1). Protein concentration was measured in MCF-7 lysate and in trypsin digest obtained from the same quantity of cancer cells. The cell lysate contained $270 \mu \mathrm{g} / \mathrm{mL}$ protein while the trypsin digest contained $\mathbf{2} \mu \mathrm{g} / \mathrm{mL}$. In order to test the immunologic properties of the trypsin digest, DC were loaded with it and used to stimulate normal human CTL in a cytotoxicity assay. Unloaded and whole-cell lysate-loaded DC were used as controls. Visual inspection of target cells during the cytotoxicity assay revealed that CTL stimulated with trypsin digest-loaded DC killed target MCF-7 cells as effectively as CTL stimulated with lysate-loaded DC (Fig. 2c, 2c',2d, 2d'). Control target cells without CTL or incubated with CTL stimulated with 

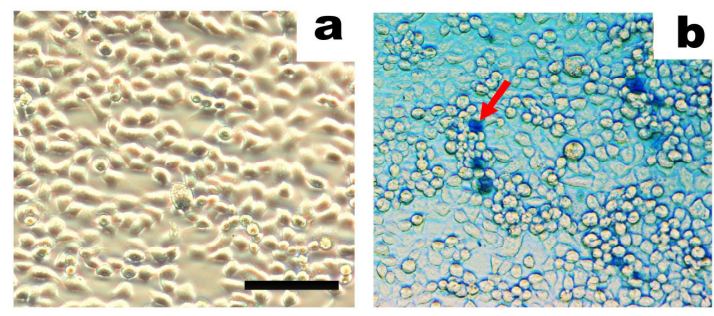

Figure 1: In situ trypan blue staining of monolayer MCF-7 cell cultures before (a) and after (b) cell treatment with trypsin. Dye stained dead cells (see arrow). Images were made by using inverted phase contrast microscope (scale bar: 50 $\mu \mathrm{m})$

unloaded DC showed progressive growth (Fig. 2a, 2a', 2b. 2b').

In the cytotoxicity assay, CTL stimulated by DC were incubated with MCF-7 cell at varying CTL-to-target cells ratios and surviving tumor cells were counted daily by trypan blue exclusion (Fig. 3). CTL killed MCF-7 cells in dosedependent manner. The CTL:MCF-7 cell ratios tested here covered a wide range of CTL activity, from low (1:1) to high (8:1). At the highest ratio, CTL killed all MCF-7 cells. The cytotoxic activity of CTL after stimulation with DC that were loaded with the trypsin digest was better (killed 10$40 \%$ more target cells; $p<0.05$ ) than CTL stimulated with DC that were loaded with lysate. This phenomenon was observed for all CTL:MCF-7 ratios.

Cytotoxic activity was not observed when unstimulated PBMC were incubated with MCF-7 cells at two different ratios $(4: 1,8: 1)$. In these cases, the MCF-7 proliferated $5 \times 10^{5}$ cells per well after 72 h. This is identical to MCF-7 cells cultured in the absence of CTL altogether (Fig. 3).

\section{Discussion}

Anti-tumor vaccines are effective because molecules on the surface of tumor cells are distinct from those presented on untransformed cells. Surface antigens are excellent targets for immune-mediated anti-cancer therapies due to their accessibility to antibodies and cytotoxic cells. Intracellular antigens are sequestered within cells and therefore less useful as antigens for vaccination. Using proteases to collect tumor-surfaceassociated antigens gives promise to generating anti-cancer immunotherapeutics by exploiting the anti-tumor response of the immune system. This approach to generate anti-cancer antigens makes sense only if treatment of cells with proteases results in low cell death rates. This is necessary to avoid contaminating the collected surface antigens by the intracellular contents released from dead cells. Figure 1 shows that selected conditions for the cells treatment with trypsin results in a cell death rate $<0.5 \%$. Thus, treating live cells with proteases allows collection of antigenic targets directly from cell surface, and prevents contamination by undesired intracellular content.

From the results of protein concentration measurements, the protein content of the trypsin digest is $0.7 \%$ from the protein content of the cell lysate. The low level of antigens in trypsin digest may

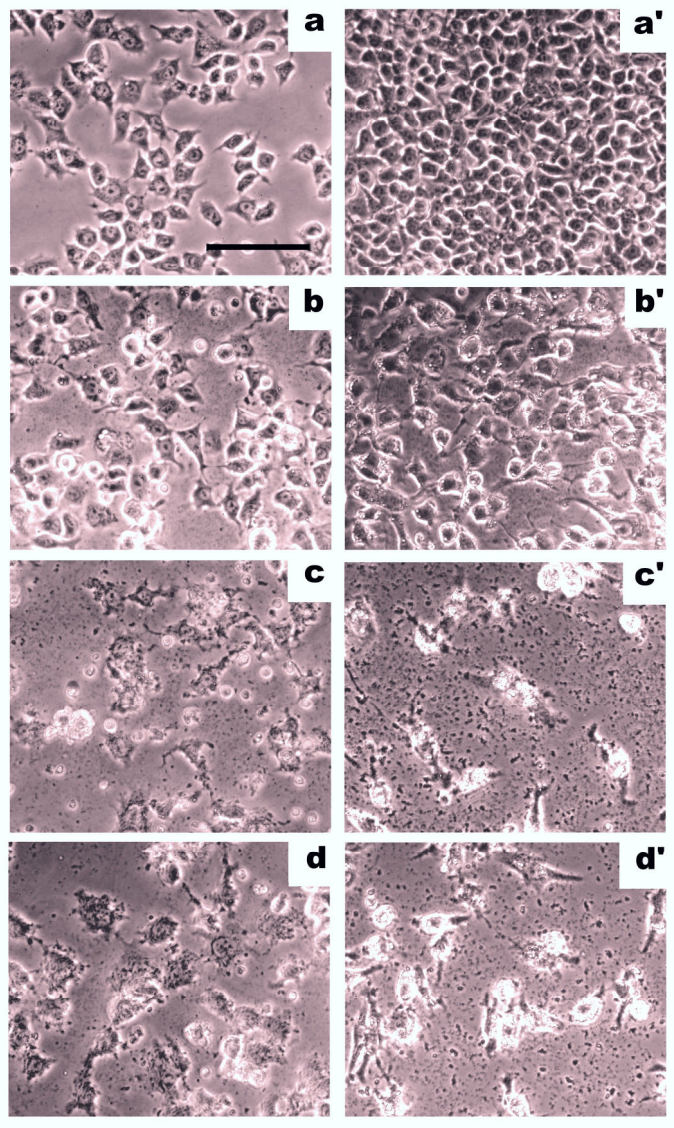

Figure 2: CTL mediated lysis of MCF-7 cells during cytotoxicity assay.

Target MCF-7 cells were seeded at $5 \times 10^{4}$ cells per well in 48-well tissue culture plates, and then effector CTL were added at CTL:MCF-7 ratio $4: 1$. On following $(a-d)$ or 3rd $\left(a^{\prime}-d^{\prime}\right)$ day, target cells were carefully washed with HBSS and photographed on an inverted phase contrast microscope (scale bar: $50 \mu \mathrm{m})$. (a, a') MCF-7 cells grown alone. ( $\left.b, b^{\prime}\right)$ MCF-7 cells incubated with CTL that had been stimulated with unloaded DC. (c, c') MCF-7 cells incubated with CTL that had been stimulated with lysate-loaded DC. (d, $d^{\prime}$ ) MCF-7 cells incubated with CTL that had been stimulated with trypsin digest-loaded DC 
be explained in the following manner. Proteases only cleave extracellular portions of plasma-membrane-associated proteins that are accessible to the protease, which in total justifies the observed low level of proteins in trypsin digest.

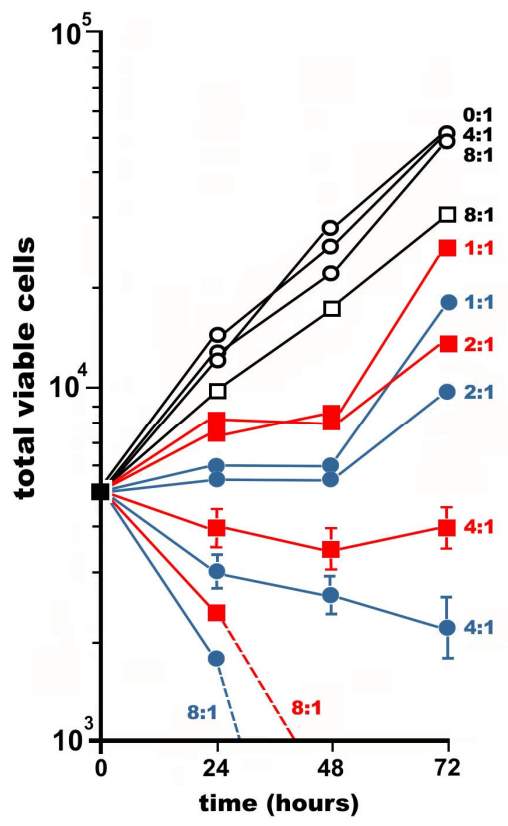

Figure 3: Cytotoxicity of effector CTL against MCF-7 cells.

Target MCF-7 cells were seeded at $5 \times 10^{4}$ cells per well in 48-well tissue culture plates, and then effector CTL were added at CTL:MCF-7 ratios ranging from $1: 1$ to $8: 1$. A ratio of $0: 1$ indicates growth of MCF-7 cells to which effector CTL were not added. $(\bullet)$ - MCF-7 cells incubated with CTL that had been stimulated with trypsin digest-loaded DCs. ( $₫$ ) - MCF-7 cells incubated with CTL that had been stimulated with lysate-loaded DCs. (口) - MCF-7 cells incubated with CTL that had been stimulated with unloaded DCs. (o) - MCF-7 cells grown either alone, or with unstimulated PBMC. Points represent the mean value of 3 identical measurements. As an example, the standard deviation for the 4:1 ratio is shown (similar values for standard deviation were obtained using other CTL:MCF-7 ratios)

To confirm that trypsin digest contains the essential antigens for vaccination, the immunogenic properties of trypsin digest and cell lysate were compared. In an in vitro model of human anti-tumor therapies, human CTL were incubated with tumor cell targets at varying CTL-to-tumor cell ratios. Microscopic photographs (Fig. 2) and results of cytotoxicity assays (Fig. 3) show that DC loaded with the trypsin digest stimulated the cytotoxic actions of CTL just as effectively as DC loaded with MCF-7 lysate. Therefore, we assumed that despite the trypsin digest containing less protein than the lysate, it had the same ability to induce cytotoxic activity in CTL. This is due to the necessary antigens being present in the trypsin digest that is free of intracellular substances. This is a proof-ofconcept, that the treatment of live tumor cells with proteases leads to the release of antigenic targets from cells that is sufficient to induce anti-tumor immune responses in vitro.

\section{Conclusions}

Further characterization of trypsin digests, such as the choice of adjuvant and schedule, is required for vaccine development. However, the diversity of native tumor-associated antigens, low levels of undesirable antigens, and purity regarding dangerous intracellular agents, demonstrates the potential for trypsin digests as anti-cancer vaccine candidates.

\section{Acknowledgement}

We would like to thank and ZAO BioBohemia (Moscow, Russia) for financial support.

\section{Conflict of Interests}

Authors declare that they have competing financial interest. Patents value may be affected by publication.

\section{References}

1. [No authors listed]. Trial watch : Progress for Phase III cancer vaccines. Nat Rev Drug Discov. 2008;7(12):9667.

2. Copier J, Dalgleish A. Overview of tumor cell-based vaccines. Int Rev Immunol. 2006;25(5-6):297-319.

3. Levine MM, Sztein MB. Vaccine development strategies for improving immunization: the role of modern immunology. Nat Immunol. 2004;5(5):460-4.

4. Emens LA. Roadmap to a better therapeutic tumor vaccine. Int Rev Immunol. 2006;25(5-6):415-43.

5. Dalgleish A, Pandha H. Tumor antigens as surrogate markers and targets for therapy and vaccines. Adv Cancer Res. 2007;96:175-90. 
6. Fukuda M. Possible roles of tumorassociated carbohydrate antigens. Cancer Res. 1996;56(10):2237-44.

7. Hakomori S. Tumor-associated carbohydrate antigens defining tumor malignancy: basis for development of anti-cancer vaccines. Adv Exp Med Biol. 2001;491:369-402.

8. Balashova EE, Lokhov PG. Proteolytically-cleaved fragments of cell surface proteins stimulate a cytotoxic immune response against tumoractivated endothelial cells in vitro. J Cancer Sci Ther. 2010;2:126-31.

9. Hudson L, Hay FC, editors. Practical Immunology. Oxford: Blackwell Scientific Publications; 1980.

10. Nestle FO, Alijagic S, Gilliet M, Sun $Y$, Grabbe $S$, Dummer $R$, et al. Vaccination of melanoma patients with peptide- or tumor lysate-pulsed dendritic cells. Nat Med. 1998;4(3):328-32.

11. Romani N, Gruner S, Brang D, Kampgen $E$, Lenz A, Trockenbacher B, et al. Proliferating dendritic cell progenitors in human blood. J Exp Med. 1994;180(1):83-93.

12. John J, Dalgleish A, Melcher A, Pandha H. Cryopreserved dendritic cells for intratumoral immunotherapy do not require re-culture prior to human vaccination. J Immunol Methods. 2005;299(1-2):37-46.

13. Yun CO, Nolan KF, Beecham EJ, Reisfeld RA, Junghans RP. Targeting of $T$ lymphocytes to melanoma cells through chimeric anti-GD3 immunoglobulin T-cell receptors. Neoplasia. 2000;2:449-59. 